\title{
Impact of CDI05 Flow-Cytometric Expression on Childhood B-Acute Lymphoblastic Leukemia
}

This article was published in the following Dove Press journal:

Journal of Blood Medicine

\author{
Rasha A Elkholy (D) \\ Mohamed H Fouda' \\ Eslam E Elhawary ${ }^{2}$ \\ Reem A Elkholy ${ }^{3}$ \\ Ola A Elshora' \\ 'Clinical Pathology Department, Faculty \\ of Medicine, Tanta University, Tanta, \\ Egypt; ${ }^{2}$ Pediatrics Department, Faculty of \\ Medicine, Tanta University, Tanta, Egypt; \\ ${ }^{3}$ Pharmacology Department, Faculty of \\ Medicine, Tanta University, Tanta, Egypt
}

Correspondence: Rasha A Elkholy Department of Clinical Pathology, Faculty of Medicine, Tanta University, Aljaysh Street, Tanta, 3195I, Egypt

Tel +20 I224489930

Fax + 2 040/3407734

Email rashaelkholy26@gmail.com
Background: CD105 (Endoglin) is a receptor of the transforming growth factor-Beta (TGF$\beta$ ) superfamily. It is expressed in angiogenic endothelial cells and is considered a powerful marker of angiogenesis and a potential main player in the pathogenesis of vascular diseases as well as tumor progression. CD105 expression was correlated with poor prognosis in many types of solid malignancies, however, its influence on hematological neoplasms is still an area of interest.

Purpose: To assess the flow-cytometric expression of CD105 in childhood B-acute lymphoblastic leukemia (B-ALL) and its relation to disease response after the induction chemotherapy.

Subjects and Methods: Eighty children newly diagnosed with B-ALL were screened for flow-cytometric expression of CD105 at time of diagnosis, then they were followed up to detect their response to induction therapy.

Results: CD105 was expressed in $41.2 \%$ of B-ALL patients. Higher expression of CD105 was observed in high and very high-risk groups. The multivariate analysis considered CD105 positivity as an independent prognostic marker for response to induction therapy. Values higher than 2.5 Specific fluorescence indices (SFIs) and 35\% expression were sensitive predictors to induction failure.

Conclusion: CD105 can be considered as a potential prognostic marker for the detection of response to induction therapy in childhood B-ALL, and it can serve to optimize treatment decisions.

Keywords: B-acute lymphoblastic leukemia, endoglin, CD105

\section{Introduction}

Acute lymphoblastic leukemia (ALL) is a malignant disorder representing clonal expansion and arrest of maturation of lymphoid progenitor cells in the bone marrow, blood, and extramedullary sites. ${ }^{1}$ The incidence of ALL generally follows a bimodal distribution, with its first peak occurring in childhood and the second around the age of $50 .^{2}$

With proper risk stratification, ALL responds well to chemotherapy, yet early mortality during the induction phase of chemotherapy is not uncommon. Besides, the high relapse rate remains a major problem. ${ }^{3}$ Therefore, identification of new prognostic markers will not only aid in increasing the accuracy of patients' risk stratification but also will minimize the chances of relapse by optimizing therapy at the early stages of treatment. ${ }^{4}$

Flow-cytometry immunophenotyping is considered a potent technology used to identify cell membrane antigens. ${ }^{5}$ The identification of surface antigens on 
leukemic cells is essential for the assignment of the proper treatment plan and is also valuable for assessing prognosis and searching for applicable markers to detect minimal residual disease. ${ }^{6}$

CD105 (Endoglin) is a homodimeric transmembrane co-receptor that interacts with transforming growth factorBeta (TGF- $\beta$ ) receptors type I and III, consequently adjusting angiogenesis by regulating proliferation, differentiation, and endothelial cell migration. ${ }^{7}$ It is expressed on the surface of endothelial cells, stromal cells, melanocytes, and different hematopoietic cells. ${ }^{8-10}$ It is highly expressed as well on the surface of activated vascular endothelial cells but not or weakly expressed on the normal quiescent vessels. Therefore, it has been proposed as a marker of angiogenesis. ${ }^{9}$ In contrast, lack of CD105 expression is diagnostic for hereditary hemorrhagic telangiectasia type -1 , a disease characterized by multiple vascular malformations. ${ }^{11}$

Buhring et $\mathrm{al}^{12}$ reported that CD105 was not detectable on normal bone marrow $\mathrm{CD} 34$ positive hemopoietic precursor cells, mature T, B, natural killer, and myeloid cells, but was instead present on a subset of glycophorin A-positive mononuclear cells. In a subsequent study, Rokhlin et $\mathrm{al}^{13}$ demonstrated the existence of two CD105 positive populations in bone marrow: B-lineage precursor cells and pro-erythroblasts. The expression of CD105 on hemopoietic precursor cells serves as a mediator affecting quiescence and enables long-term repopulation. ${ }^{14}$

Regarding hematological malignancies, CD105 expression was reported in myelodysplastic syndrome, ${ }^{15}$ ALL, ${ }^{16,17}$ and acute myeloid leukemia (AML). ${ }^{16-19}$ CD105 positive blast cells exhibit higher leukemogenic activity when compared to their negative counterpart. ${ }^{20}$

Cosimato, and his colleagues, ${ }^{16}$ extensively studied the expression of CD105 on blast cells of acute leukemia patients and reported that CD105 was expressed in the majority of B-ALL cases, and only the most immature types expressed this antigen, as it was consistently absent in "Burkitt-like" mature B-ALL without referring to its effect on patient's prognosis. Also, Poręba et al ${ }^{17}$ reported that CD105 expression in patients with ALL was limited to a small group of patients and needs to be confirmed on a larger group. So, adequate information about its prognostic impact in B-ALL is still worth further evaluation.

The current work aimed to assess the CD105 flowcytometric expression in B-ALL pediatric patients and its relation to disease response after the induction phase of chemotherapy.

\section{Subjects and Methods}

The current study was carried out on 80 children newly diagnosed with B-ALL referred to Hematology/Oncology Unit, Pediatric Department, Tanta University Hospitals, and Pediatric Oncology Unit, Tanta Cancer Center from June 2017 to August 2020.

Cases were diagnosed based on clinical presentation, complete blood count (CBC), bone marrow (BM) examination, morphological and cytochemical smears as well as immunophenotyping.

Risk stratification was done according to Children's Oncology Group (COG) protocols. ${ }^{21}$ Cases were classified into three groups; standard-risk group (54 cases, 67.5\%), high-risk group (17 cases, $21.25 \%$ ), and very high-risk group (9 cases, $11.25 \%$ ).

Initial investigations included; automated CBC on ERMA PCE-210N cell counter (Tokyo, Japan) with an examination of Giemsa-stained smears, Liver and renal function tests, and lactate dehydrogenase enzyme (LDH) on a fully automated chemistry analyzer (Konelab Prime 60i, Thermo-scientific, Vantaa, Finland), erythrocyte sedimentation rate (ESR), and cerebrospinal fluid (CSF) cytological examination. BM aspiration samples were evaluated through Giemsa-stained smears, and acute leukemia was diagnosed by the presence of $\geq 20 \%$ blast cells. Philadelphia ( $\mathrm{Ph})$ chromosome was detected by fluorescence in-situ hybridization (FISH).

Immunophenotyping analysis was done on BM samples collected into EDTA-containing tubes using the fourcolor flow cytometry Becton Dickinson (BD) FACS Calibur instrument (Becton Dickinson, San Diego, California, USA), using the Cell Quest software (Becton Dickinson, version 3, verify software House Topsham, ME, USA). Before each run, calibrated beads provided by the manufacture were used to adjust the compensation of different fluorochromes. Mouse isotopic controls were used as negative controls to exclude autofluorescence. At least 10.000 events/tubes were acquired for each analysis. Blast cells were identified based on dim/intermediate CD45 Peridinin Chlorophyll Protein Complex (Per-CP) expression versus log side scatter characteristics (CD45/ SSC gating strategy). The gated fluorescence dot plot was evaluated for positive cells using cursor position from the dot plot of isotypic controls. The internal negative control was checked using normal cells in the sample that lacked the antigen, while the internal positive control was checked using the normal cells in the sample that expressed the antigen. 
B-ALL was diagnosed using the acute leukemia panel that included the following combinations of surface markers: CD45/CD14/CD117/CD34, CD45/HLA-DR/CD10/ CD38， CD45/CD64/CD19/CD20， CD45/CD7/CD33， $\mathrm{CD} 45 / \mathrm{CD} 2 / \mathrm{CD} 13$, and the cytoplasmic markers anti TDT/anti MPO, anti CD79a and anti cyt $\mu$, markers were supplied by Becton Dickinson (BD biosciences, Mountain View, California). B-ALL blast cells were identified by positive expression of CD19 in addition to CD10 and/or CD79a.

Immunophenotyping analysis of CD105 using CD105 fluorescein isothiocyanate (FITC) labeled monoclonal antibody, supplied by BD biosciences, Catalog number 561443 Clone 266 (RUO). The total leucocytic count (TLC) was adjusted to $10^{6}$ cells/tube. Cells were incubated with $5 \mu$ of CD45 Per-CP, and $10 \mu$ of CD105 FITC in the dark, at room temperature, for $25 \mathrm{~min}$. Red blood cells were then lysed with $1 \mathrm{~mL}$ of BD FACS lysing solution for $20 \mathrm{~min}$ before centrifugation, the cells were then washed twice with $0.5 \mathrm{~mL}$ of phosphate buffer saline (PBS), and suspended in $300 \mu \mathrm{L}$ of PBS to be ready for the flow cytometer acquisition. Blast cells were selectively gated using CD45/SSC strategy, and the percent of blast cells expressing CD105 was determined within this population using a cutoff value for positivity $>5 \%{ }^{17}$ Specific fluorescence indices (SFIs) were calculated by dividing median fluorescence intensity (MFI) of CD105 by MFI of negative isotype control, positive expression was defined as SFIs $>1.5^{19}$ (Figures 1 and 2).

After being fully investigated at diagnosis, all the patients received induction chemotherapy, according to the protocol adopted by the treating centers; the Modified St Jude Children's Research Hospital (SJCRH) Total Therapy XV Protocol. ${ }^{22}$ At the end of induction therapy on day 28 , all patients were reevaluated by $\mathrm{CBC}$ and $\mathrm{BM}$ samples. Remission was identified by the absence of peripheral blood blasts and BM blast cells less than 5\%. Refractoriness to therapy was defined by the presence of greater than 5\% BM blasts and/or CNS infiltration by leukemic cells. Those who did not achieve complete remission were reassigned to more intensified treatment protocols. $^{22}$

\section{Statistical Analysis}

Data were analyzed using IBM SPSS software package version 20.0. (Armonk, NY: IBM Corp). The Kolmogorov-Smirnov test was used to verify the normality of the distribution of variables. Comparisons between groups for categorical variables were assessed using the Chi-square test [Fisher or Monte Carlo (MC)]. Student's $t$-test was used to compare two groups of normally distributed quantitative variables while the Mann Whitney test was used to compare two groups of non-normally distributed quantitative variables. For nonnormally distributed quantitative variables more than two groups Kruskal Wallis test was used and followed by the Post Hoc test (Dunn's for multiple comparisons test). Pairwise comparison Spearman coefficient was used to correlate between quantitative variables. Univariate and multivariate logistic regression analyses were used to determine the related co-variables associated with poor response to induction therapy. The Receiver operating characteristic curve (ROC) was used to determine the diagnostic characteristics of the marker. The optimal cutoff value was assessed via the Youden index. The significance of obtained results was judged at the $\leq 0.05$ level. ${ }^{23}$

\section{Results}

This prospective study was conducted on eighty children newly diagnosed with B-ALL. They were 50 boys (62.5\%) and 30 girls $(37.5 \%)$ with a male to female ratio of $1.6: 1$, their ages ranged from 1 to 15 years with a median value 7.3 years.

At diagnosis, the mean and standard deviation (SD) of the hemoglobin $(\mathrm{Hb})$ level was $7.9 \pm 1.8(\mathrm{gm} / \mathrm{dl})$, blast percentage in peripheral blood (P.B) $38 \pm 23.3$ (\%), and ESR $82 \pm 15.3(\mathrm{~m} / \mathrm{h})$. The median and range for the TLC was $20(1-110) \times 10^{3} / \mathrm{cmm}$, platelets count $65(10-260)$ $\mathrm{x} 10^{3} / \mathrm{cmm}$, blast percentage in bone marrow $82.5(50-98)$ \%, LDH level 822 (340-3200) IU/L.

As regard immunophenotyping results, CD19 was expressed in all cases $80 / 80(100 \%)$ of B-ALL, CD10 in $66 / 80(82.5 \%)$ cases, CD34 in 71/80 (88.7\%) cases, cytoplasmic $\mu$ in 12/80 (15\%) cases, CD20 in $9 / 80(11.2 \%)$ cases, with aberrant expression of CD33 and CD13 in 6/80 (7.5\%) and 15/80 (18.7\%) cases respectively. Based on these data, patients were classified according to EGIL classification as follows: pro-B-ALL (5 cases), common B-ALL (63 cases), pre-B-ALL (3 cases), and mature B-ALL ( 9 cases).

Positive expression of CD105 on BM samples was detected in $33 / 80(41.2 \%)$ patients, and it ranged from $8-90 \%, 2-8$ SFIs with a median value of $36 \%$ and 3 SFIs respectively, while negative expression was detected in $47 / 80(58.8 \%)$ patients, and ranged from $0.5-5 \%$, 

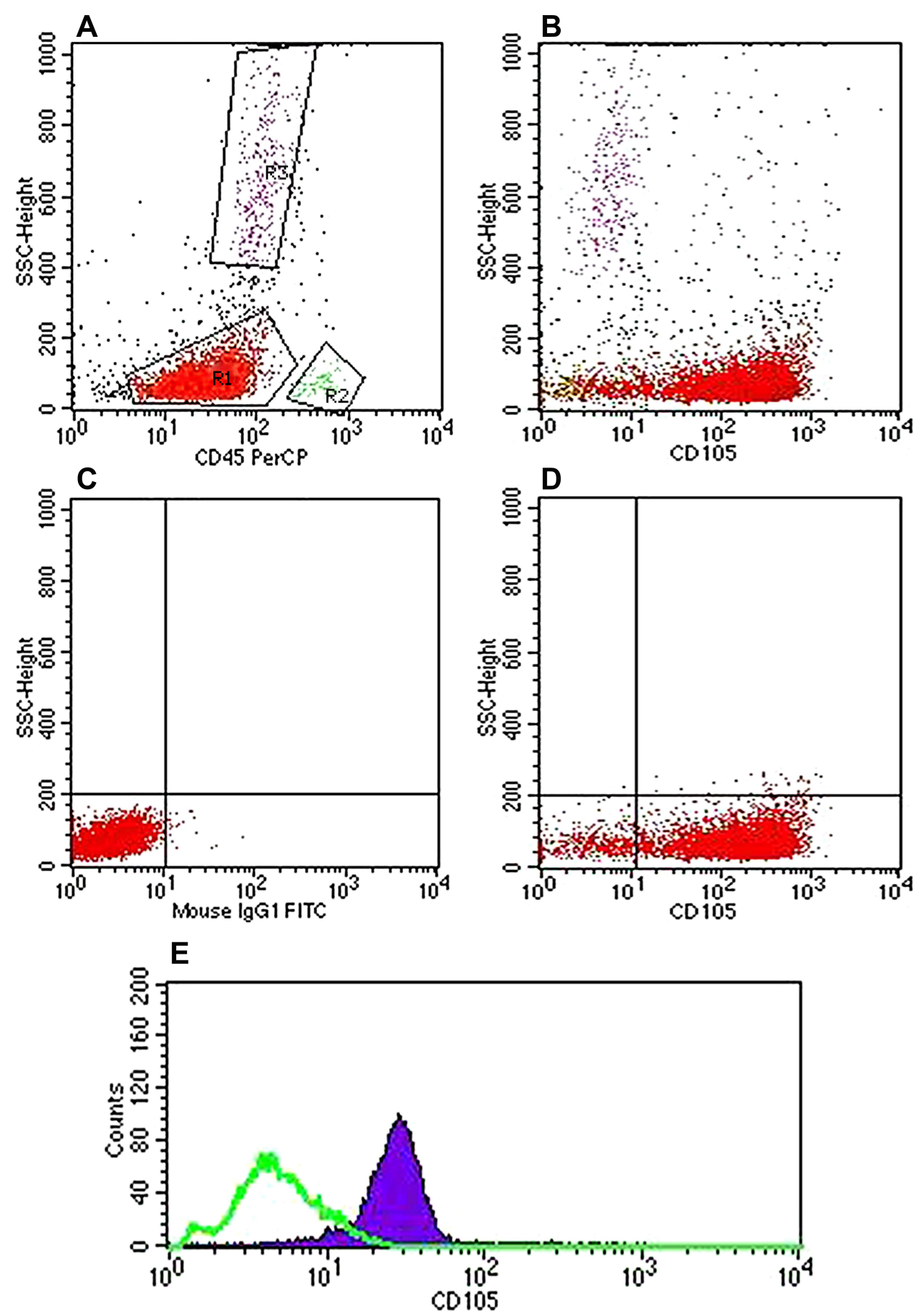

Figure I Flow cytometry analysis of CDI05 positive case. (A) Dot plot showed gating using CD45/SSC strategy, blast cells (RI), normal lymphocytes (R2) and granulocytes (R3). (B) Scatter dot plot showing blast cells positive for CDI05 expression in combination with normal lymphocytes and granulocytes negative for CDI05 expression. (C) Dot plot showing the mouse IgGI isotypic negative control. (D) Dot plot showing positive CDI05 expression on gated blast cells. (E) Histogram showing positive CDI05 (solid violet curve) versus negative control (green colored curve).

0.5-1.4 SFIs with a median value of $3 \%$ and 1 SFIs respectively.

CD105 expression in B-ALL subtypes was positive in (3/5) Pro B-ALL, (29/63) common B-ALL, (1/3) Pre B-ALL, while it was absent (0/9) in all cases of "Burkittlike" mature B-ALL.
We found variations between CD105 percentage, SFIs and the patients' risk stratification, with statistically significant difference between the standard-risk and the very high-risk groups (P-value $=0.045$, and 0.035 respectively) as shown in (Table 1). No statistically significant difference was observed between the CD105 positive and the 

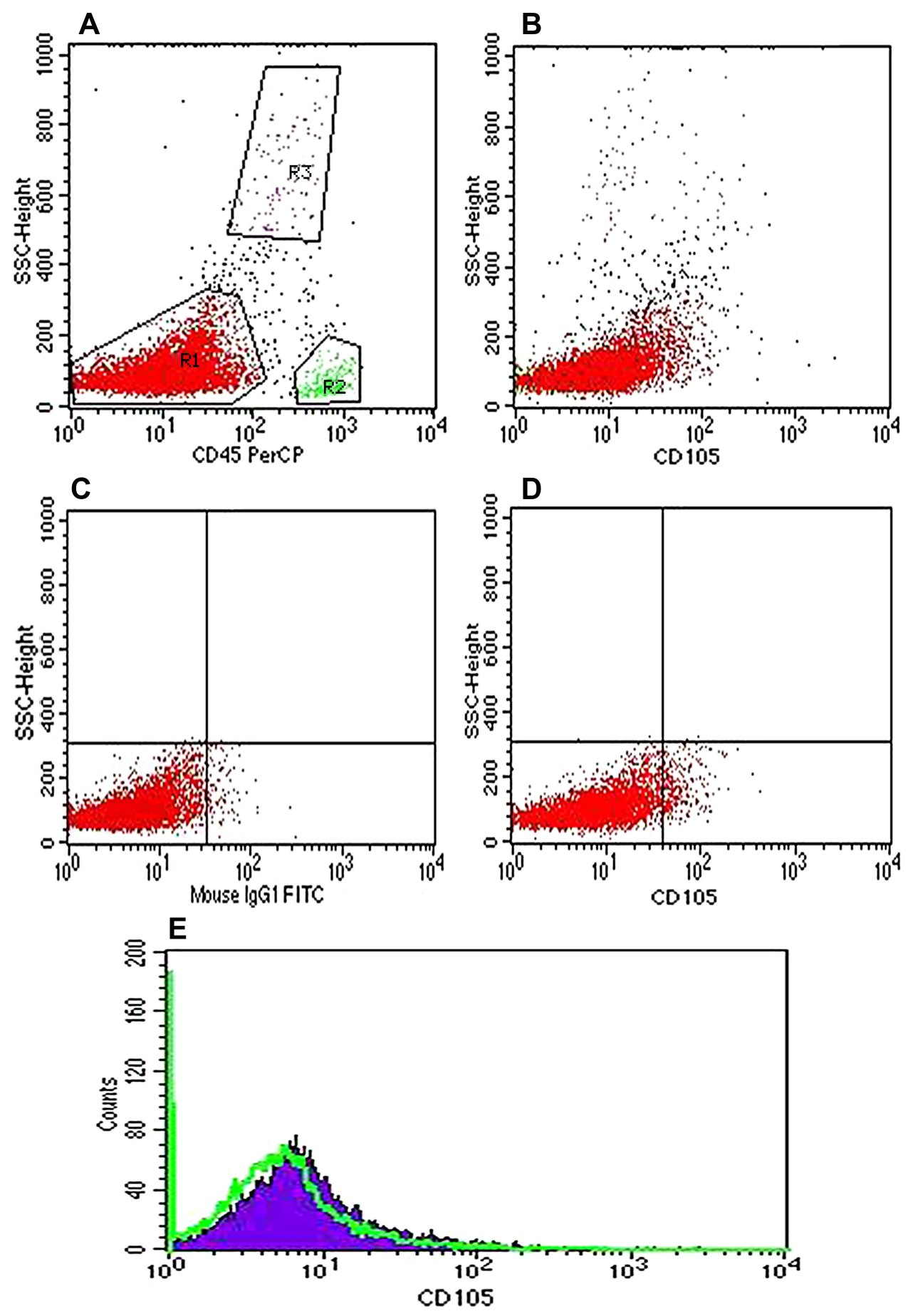

Figure 2 Flow cytometry of analysis of CDI05 negative case. (A) Dot plot showed gating using CD45/SSC strategy, blast cells (RI), normal lymphocytes (R2) and granulocytes (R3). (B) Scatter dot plot showing blast cells, normal lymphocytes and granulocytes all negative for CDI05 expression. (C) Dot plot showing the mouse lgGI isotypic negative control. (D) Dot plot showing negative CDI05 expression on gated blast cells. (E) Histogram showing negative CDI05 (solid violet curve) versus negative control (green colored curve).

negative groups as regard clinical and laboratory parameters (P-value $>0.05)$ (Table 2).

CD105 expression had a weak positive correlation $(<0.3)$ with age and TLC, LDH, and a weak negative correlation $(<0.3)$ with CD20 as presented in (Table 3).
At follow up, 15 out of 80 patients were excluded from the statistical analysis (9 patients chose to complete induction at other centers, 3 of them were among the mature B-ALL group, the other 6 mature B-ALL patients were intentionally excluded as they received a different treatment protocol). 
Table I The Pattern and Range of CDI05 Expression in B-ALL Cases as Regards Risk Stratification

\begin{tabular}{|l|l|l|l|l|l|}
\hline & Standard Risk (54 Patients) & High Risk (17 Patients) & Very-High (9 Patients) & P-value & Post Hoc Test \\
\hline $\begin{array}{l}\text { CD 105 SFIs } \\
\text { Median (Min.- Max.) }\end{array}$ & $1.2(0.5-6)$ & $1.3(0.5-6)$ & $6(1-8)$ & $0.035 *$ & $P_{1}: 0.239, P_{2}: 0.013^{*}, P_{3}: 0.168$ \\
\hline $\begin{array}{l}\text { CD 105\% } \\
\text { Median (Min. - Max.) }\end{array}$ & $4(0.5-60)$ & $4(2-60)$ & $50(1-90)$ & $0.045 *$ & $P_{1}: 0.169, P_{2}: 0.022^{*}, P_{3}: 0.282$ \\
\hline
\end{tabular}

Notes: *Significant at $\mathrm{P} \leq 0.05$. $\mathrm{P}_{1}$ : $\mathrm{P}$-value for comparing between standard and high risk. $\mathrm{P}_{2}$ : $\mathrm{P}$-value for the association between standard and very high. $\mathrm{P}_{3}$ : $\mathrm{P}$-value for the association between high risk and very high.

Abbreviation: SFIs, specific fluorescence indices.

The remaining 65 cases were followed till the end of and 15 had CD105 positive expression. 10/65 cases induction. Remission was achieved by $51 / 65$ (78.4\%) (15.4\%) were refractory to the first induction cycle, one cases; 36 of them had a negative expression of CD105 of them was CD105 negative while and 9 were CD105

Table 2 The Impact of CDI05 Expression Pattern on B-ALL Patient's Characteristics

\begin{tabular}{|c|c|c|c|}
\hline Parameters & CD 105 Negative Group (47 Patients) & CD 105 Positive Group (33 Patients) & P-value \\
\hline \multicolumn{4}{|l|}{ Age (years) } \\
\hline Median (Min. - Max.) & $6.5(1.5-14)$ & $8.5(1-15)$ & 0.109 \\
\hline \multicolumn{4}{|l|}{ Hemoglobin (gm/dl) } \\
\hline Mean \pm SD & $8.1 \pm 1.6$ & $7.7 \pm 2$ & 0.394 \\
\hline \multicolumn{4}{|l|}{ Total leucocytic count $\left(\times 10^{3} / \mathrm{cmm}\right)$} \\
\hline Median (Min. - Max.) & $18(1-110)$ & $30(1.7-96)$ & 0.052 \\
\hline \multicolumn{4}{|l|}{ Platelets count $\left(\times 10^{3} / \mathrm{cmm}\right)$} \\
\hline Median (Min. - Max.) & $57(10-260)$ & $70(15-185)$ & 0.384 \\
\hline \multicolumn{4}{|l|}{ Peripheral blood blasts (\%) } \\
\hline Mean \pm SD. & $36.2 \pm 20.8$ & $40.6 \pm 26.7$ & 0.324 \\
\hline \multicolumn{4}{|l|}{ Bone marrow blasts (\%) } \\
\hline Median (Min. - Max.) & $80(50-98)$ & $90(59-98)$ & 0.634 \\
\hline \multicolumn{4}{|l|}{ Lactate dehydrogenase (IU/L) } \\
\hline Median (Min. - Max.) & $787(340-2300)$ & $900(444-3200)$ & 0.095 \\
\hline \multicolumn{4}{|l|}{ Erythrocyte sedimentation rate $(\mathrm{m} / \mathrm{h})$} \\
\hline Mean \pm SD & $82.6 \pm 15.4$ & $81.1 \pm 15.3$ & 0.667 \\
\hline \multicolumn{4}{|l|}{ Sex } \\
\hline Male & $32(68.1 \%)$ & $18(54.5 \%)$ & ${ }^{\mathrm{FE}} \mathrm{p}=0.218$ \\
\hline Female & $15(31.9 \%)$ & 15 (45.5\%) & \\
\hline \multicolumn{4}{|l|}{ Central nervous system infiltration } \\
\hline Absent & 45 (95.7\%) & $30(90.9 \%)$ & ${ }^{F E}{ }_{p}=0.644$ \\
\hline Present & $2(4.3 \%)$ & $3(9.1 \%)$ & \\
\hline \multicolumn{4}{|l|}{ Philadelphia chromosome } \\
\hline Negative & $46(97.9 \%)$ & $28(84.8 \%)$ & ${ }^{F E} \mathrm{p}=0.077$ \\
\hline Positive & I (2.I\%) & $5(15.2 \%)$ & \\
\hline \multicolumn{4}{|l|}{ Testicular infiltration } \\
\hline Present & $2(4.3 \%)$ & $4(12.1 \%)$ & ${ }^{F E} \mathrm{p}=0.224$ \\
\hline Absent & 45 (95.7\%) & $29(87.9 \%)$ & \\
\hline
\end{tabular}

Abbreviation: FE, Fisher exact. 
Table 3 Correlation Between CDI05 SFIs and CDI05\% with Different Parameters

\begin{tabular}{|c|c|c|c|c|}
\hline & \multicolumn{2}{|c|}{ CDI05 SFIs } & \multicolumn{2}{|c|}{ CDI05\% } \\
\hline & $\mathbf{r}_{\mathbf{s}}$ & P-value & $\mathbf{r}_{\mathbf{s}}$ & P-value \\
\hline Age (years) & 0.298 & $0.007 *$ & 0.293 & $0.008 *$ \\
\hline Hemoglobin (gm/dl) & -0.151 & 0.181 & -0.105 & 0.354 \\
\hline Total leucocytic count $\left(\times 10^{3} / \mathrm{cmm}\right)$ & 0.261 & $0.019 *$ & 0.230 & $0.040 *$ \\
\hline Platelets count $\left(\times 10^{3} / \mathrm{cmm}\right)$ & -0.039 & 0.731 & -0.025 & 0.824 \\
\hline Peripheral blood blasts (\%) & 0.059 & 0.606 & 0.081 & 0.473 \\
\hline Bone marrow blasts (\%) & 0.113 & 0.320 & 0.189 & 0.092 \\
\hline Lactate dehydrogenase (IU/L) & 0.236 & $0.035^{*}$ & 0.199 & 0.077 \\
\hline Erythrocyte sedimentation rate $(\mathrm{m} / \mathrm{h})$ & 0.018 & 0.871 & -0.036 & 0.754 \\
\hline CDI9 (\%) & 0.041 & 0.721 & 0.018 & 0.874 \\
\hline CD20 (\%) & -0.232 & $0.038 *$ & -0.179 & 0.113 \\
\hline
\end{tabular}

Notes: *Significant at $\mathrm{P} \leq 0.05$. $\mathrm{r}_{\mathrm{s}}$ : Spearman coefficient.

Abbreviation: SFIs, specific fluorescence indices.

positive expression. Four children died during the induction stage, one of them was CD105 negative and the other three were CD105 positive. Deaths were due to be sepsis with severe neutropenia in three children and intracranial hemorrhage with severe thrombocytopenia in one child.

The difference between the CD105 positive and the negative group regarding the response to therapy was highly significant $\left(X^{2} 14.383 *{ }^{\mathrm{MC}} \mathrm{P}\right.$-value $\left.<0.001\right)$.

To establish the effect of CD105 expression on response to induction therapy, logistic regression analyses were performed to detect the related covariables that can be associated with poor response to induction therapy. In univariate analysis, $\mathrm{Ph}$ chromosome positivity, and CD105 positivity were significantly associated with poor response to therapy (P-value $\leq 0.05$ ). In multivariate analysis, CD105 positivity remained the only significant independent factor associated with poor response to induction therapy (P-value $\leq 0.05$ ). (Table 4).

As CD105 positivity was significantly correlated to poor response to induction therapy, we used the Receiveroperating characteristic (ROC) curve analysis to establish the most suitable cutoff value after exclusion of mature B-ALL cases, missed cases, and deaths. ROC curve analysis showed that CD105 expression $>35 \%$ and SFIs $>2.5 \%$ is highly significant to differentiate between good and poor responders to induction therapy with high diagnostic efficacy (AUROC 0.948 and 0.928 respectively) (Table 5) and (Figure 3).

Table 4 Univariate and Multivariate Analysis for the Parameters Affecting Response to Induction Therapy

\begin{tabular}{|c|c|c|c|c|}
\hline & \multicolumn{2}{|r|}{ Univariate } & \multicolumn{2}{|c|}{ Multivariate } \\
\hline & P-value & OR (95\% C.I) & P-value & OR (95\% C.I) \\
\hline Age ( $>8$ versus. $\leq 8$ years) & 0.232 & $2.325(0.582-9.283)$ & & \\
\hline Sex (female versus. male) & 0.213 & $2.400(0.605-9.522)$ & & \\
\hline Hemoglobin $(>7.8 \mathrm{gm} / \mathrm{dl}$ versus. $\leq 7.8 \mathrm{gm} / \mathrm{dl})$ & 0.195 & $0.38 \mid(0.088-1.640)$ & & \\
\hline Total leucocytic count $\left(>20 \times 10^{3} / \mathrm{cmm}\right.$ versus. $\left.\leq 20 \times 10^{3} / \mathrm{cmm}\right)$ & 0.280 & $2.143(0.538-8.540)$ & & \\
\hline Platelets count $\left(>65 \times 10^{3} / \mathrm{cmm}\right.$ versus $\left.\leq 65 \times 10^{3} / \mathrm{cmm}\right)$ & 0.865 & I.I $25(0.290-4.366)$ & & \\
\hline Peripheral blood blasts ( $>40 \%$ versus. $\leq 40 \%$ ) & 0.385 & $1.833(0.468-7.187)$ & & \\
\hline Bone marrow blasts (> $89 \%$ versus. $\leq 89 \%$ ) & 0.161 & $2.84 \mid(0.659-12.239)$ & & \\
\hline Lactate dehydrogenase (>856 IU/L versus. $\leq 856 \mathrm{IU} / \mathrm{L})$ & 0.998 & - & & \\
\hline Erythrocyte sedimentation rate $(>85 \mathrm{~m} / \mathrm{h}$ versus. $\leq 85 \mathrm{~m} / \mathrm{h})$ & 0.683 & $0.750(0.189-2.980)$ & & \\
\hline Central nervous system infiltration (present versus. absent) & 0.635 & $1.778(0.166-19.065)$ & & \\
\hline Philadelphia chromosome (positive versus. negative) & $0.049 *$ & $12.500(1.012-154.397)$ & 0.110 & $14.820(0.54-405.82)$ \\
\hline CDIO5 (positive versus negative) & $0.005^{*}$ & $21.600(2.5 \mathrm{II}-185.80)$ & $0.007^{*}$ & $22.807(2.34-221.84)$ \\
\hline
\end{tabular}

Notes: *Significant at $\mathrm{P} \leq 0.05$. The number between brackets in columns I represent cutoff used was the median of the cases included.

Abbreviations: OR, odds ratio; C.I, confidence interval. 
Table 5 Performance (AUC, Sensitivity, Specificity) for CDI05 SFIs and CD 105\% to Discriminate Between Poor Responder $(n=10)$ and Good Responder $(\mathrm{n}=5 \mathrm{I})$

\begin{tabular}{|l|l|l|l|l|l|l|l|l|}
\hline & AUC & P-value & 95\% C.I & Cutoff & Sensitivity & Specificity & PPV & NPV \\
\hline CD 105 SFls & 0.928 & $<0.001^{*}$ & $0.800-1.057$ & $>2.5$ & 90.0 & 92.16 & 69.2 & 97.9 \\
CD 105\% & 0.948 & $<0.001^{*}$ & $0.867-1.029$ & $>35$ & 90.0 & 94.12 & 75.0 & 98.0 \\
\hline
\end{tabular}

Note: *Significant at $\mathrm{P} \leq 0.05$.

Abbreviations: AUC, area under the curve; C.I, confidence interval; NPV, negative predictive value; PPV, positive predictive value.

\section{Discussion}

The TGF- $\beta$ co-receptor CD105 plays a major role in fetal, adult, and malignant angiogenesis. Previous studies reported the CD105 expression on tumor vessels of a variety of neoplasms. ${ }^{8}$ Although, CD105 is now well known to be expressed on malignant cells in various hematopoietic malignancies, data on its prognostic relevance in especially leukemias are still not adequate. ${ }^{19}$

CD105 positivity in the current study was present in $41.2 \%$ of cases diagnosed with B-ALL, this is lower than the values presented by Cosimato et $\mathrm{al}^{16}$ who reported positive CD105 expression in $68.4 \%$ of B-ALL cases, this difference can be attributed to the relatively small number of cases involved in our study. They also found that CD105 was absent in all cases of mature B-ALL, and expression levels were higher among the high and the very high-risk groups compared to the standard group, which is similar to our findings.

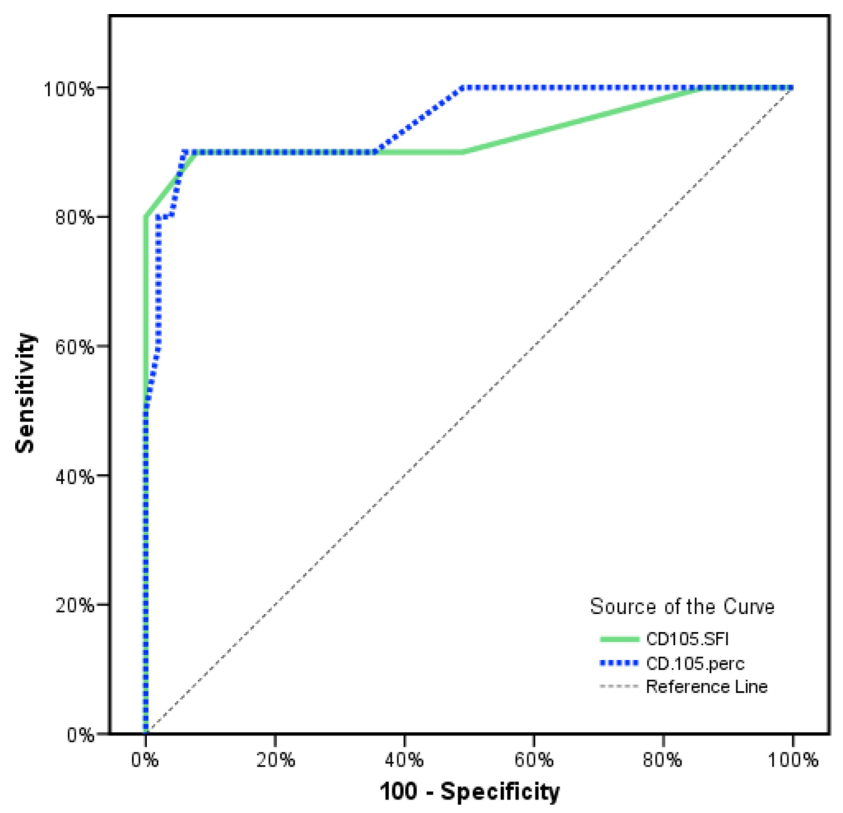

Figure 3 ROC curve for CDI05 SFIs and CDI05\%to discriminate between poor and good responder.
Although the study of Poręba et $\mathrm{al}^{17}$ reported that no statistically significant correlation was present between CD105 expression and age, gender, LDH level, blast percentage in P.B or BM, we were able to detect a weak positive correlation with age, TLC, and LDH level and a weak negative correlation between CD105 SFIs and CD20 expression.

Patients with positive CD105 expression showed a higher rate of induction failure. Moreover, the multivariate analysis showed that CD105 positivity is an independent factor that associated with poor outcome to induction therapy. This highlights the importance of CD105 as a prognostic marker in B-ALL. This is comparable to what Kauer et $\mathrm{al}^{19}$ found in their study of CD105 in AML, where they correlated it to poor outcome and failure of response to chemotherapy, and they recommended the use of CD105 expression as a prognostic marker in AML, which can help optimize follow up and treatment decisions for AML patients. They attempted to explain the inferior outcome in CD105 positive AML patients by suggesting that CD105 contributes to dysregulation of TGF- $\beta$ dependent and TGF- $\beta$ independent signaling pathways and enhances angiogenesis which gives a better chance for survival of malignant cells with increasing the risk of minimal residual disease (MDR). Also, $\mathrm{Xu}$ et $\mathrm{al}^{24}$ suggested that resistance to intensive therapy might be explained by the presence of hypoxia inducible factor 1 alpha induced multi-drug resistance transporters in $\mathrm{CD}$ 105 high AML blast cells.

To our knowledge, this is the first study that reported the impact of CD105 flow-cytometric expression on the response to induction therapy in B-ALL. Therefore, we established a cutoff value of $>35 \%$ and $>2.5$ SFIs as calculated by the ROC curve to identify patients who are at risk for induction failure that may require intensive therapy from the start to improve outcome.

Novel therapeutic targets for acute leukemia are urgently needed and successful treatment of acute leukemia remains a clinical challenge. ${ }^{25}$ For ALL, although significant progress has been made in the last decade, in 
cases of refractory or relapsed ALL, second-line chemotherapy has shown a poor effect, rarely resulting in long-term survival. ${ }^{26}$ Thus, there is a critical need for new therapeutic options. Monoclonal antibodies are promising agents because they deliver their therapeutic effects with minimal toxicity. ${ }^{27}$

CD105 is a promising target that can be used for tumor imaging and prognosis and it has therapeutic potential in patients with solid tumors and other neoplastic diseases with increased angiogenesis. ${ }^{18}$ Dourado and his colleagues, ${ }^{21}$ studied the use of monoclonal antibody TRC105 to prevent the engraftment of primary AML blasts and inhibit leukemia progression following disease establishment, but in B-ALL, TRC105 alone was ineffective due to the shedding of soluble CD105. However, in both B-ALL and AML, TRC105 synergized with reduced intensity myeloablation to inhibit leukemogenesis, indicating that TRC105 may represent a novel therapeutic option for B-ALL and AML. So, measurement of CD105 may be of great value not only to assess the prognosis but also in targeted therapy for B-ALL.

The results of our study support the important role of CD105 in leukemia progression and response to induction therapy which is one of the major contributors of B-ALL risk stratification.

The limitations of the present study included the relatively small number of patients included in this study, and the association between CD105 flow-cytometric expression and its soluble level was not investigated. Therefore, it is recommended to extend this research on a large patients cohort, investigate the coupled measurement of CD105 on blast cells and its soluble form in serum. Also, extend the follow-up duration to assess the impact of CD105 expression on the overall survival and diseasefree survival, as well as studying the stability of the marker after therapy.

\section{Conclusion}

CD105 can be considered a potential marker for the prognosis of pediatric patients with B-ALL, as patients who showed expression higher than $35 \%$ and 2.5 SFIs were at higher risk for induction failure. So, CD105 may serve to optimize treatment decisions for B-ALL patients.

\section{Data Sharing Statement}

Data can be provided on request.

\section{Ethics Approval and Consent to Participate}

The study was approved by the local ethical committee of the Faculty of Medicine, Tanta University. Institutional Review Board (IRB) for human studies (Approval Code 34107/9/20). Our study conforms to provisions of the Declaration of Helsinki. Informed written consent was obtained from one of the parents or the legal guardian accompanying the child. All personal data were kept confidential and only scientific data are available for publication.

\section{Acknowledgments}

We would like to thank cordially and express our deepest gratitude and appreciation to the team of Pediatric Oncology Unit, Tanta Cancer Institute for their cooperation, support, and follow-up of cases.

\section{Author Contributions}

All authors made essential contributions to study design, analysis and interpretation of data; took part in drafting the article and revising it critically for important intellectual content; agreed to submit to the current journal, gave final approval of the version to be published, and agree to be accountable for all aspects of the work.

\section{Funding}

This research did not receive any specific grant from funding agencies in the public, commercial, or not-forprofit sectors.

\section{Disclosure}

The authors declare that they have no conflicts of interest.

\section{References}

1. Terwilliger T, Abdul-Hay MJ. Acute lymphoblastic leukemia: a comprehensive review and 2017 update. Blood Cancer J. 2017;7 (6):e577-e577. doi:10.1038/bcj.2017.53

2. Paul S, Kantarjian H, Jabbour EJ. Adult acute lymphoblastic leukemia. Mayo Clin Proc. 2016;91(11):1645-1666. doi:10.1016/j. mayocp.2016.09.010

3. Tzortzatou-Stathopoulou F, Papadopoulou AL, Moschovi M, Botsonis A, Tsangaris GT. The low relapse rate in children with acute lymphoblastic leukemia after risk -directed therapy. J Pediatr Hematol Oncol. 2001;23(9):591-597. doi:10.1097/00043426200112000-00008

4. Rashed WM, Hamza MM, Matboli M, Salem SI. MicroRNA as a prognostic biomarker for survival in childhood acute lymphoblastic leukemia: a systematic review. Cancer Metastasis. 2019;38 (4):771-782. doi:10.1007/s10555-019-09826-0

5. Xu B, Hu C, Miao XD, et al. Immunophenotyping of 106 adult patients with acute leukemia by flow cytometry. Di Yi Jun Yi Da Xue Хие Baо. 2003;23(10):1043. 
6. Khoury H, Dalal BI, Nevill TJ. Acute myelogenous leukemia with $\mathrm{t}$ (8; 21)-identification of a specific immunophenotype. Leuk Lymphoma. 2003;44(10):1713-1718. doi:10.1080/ 1042819031000116698

7. Minhajat R, Mori D, Yamasaki F, Sugita Y, Satoh T, Tokunaga O. Organ-specific endoglin (CD105) expression in the angiogenesis of human cancers. Pathol Int. 2006;56(12):717-723. doi:10.1111/ j.1440-1827.2006.02037.x

8. Fonsatti E, Vecchio LD, Altomonte M, et al. Endoglin: an accessory component of the TGF- $\beta$-binding receptor-complex with diagnostic, prognostic, and bioimmunotherapeutic potential in human malignancies. J Cell Physiol. 2001;188(1):1-7. doi:10.1002/jcp.1095

9. Dallas NA, Samuel S, Xia L, et al. Endoglin (CD105): a marker of tumor vasculature and potential target for therapy. Clin Cancer Res. 2008;14(7):1931-1937. doi:10.1158/1078-0432.CCR-07-4478

10. Brum ST, Demasi AP, Stelini RF, Cintra ML, de Araujo VC, Soares AB. Endoglin is highly expressed in human mast cells. Appl Immunohistochem Mol Morphol. 2019;27(8):613-617. doi:10.1097/ PAI.0000000000000668

11. McAllister KA, Grogg KM, Johnson DW, et al. Endoglin, a TGF- $\beta$ binding protein of endothelial cells, is the gene for hereditary haemorrhagic telangiectasia type 1. Nat Genet. 1994;8(4):345-351. doi:10.1038/ng1294-345

12. Bühring HJ, Müller CA, Letarte M, et al. Endoglin is expressed on a subpopulation of immature erythroid cells of normal human bone marrow. Leukemia. 1991;5(10):841.

13. Rokhlin OW, Cohen MB, Kubagawa H, et al. Differential expression of endoglin on fetal and adult hematopoietic cells in human bone marrow. J Immunol. 1995;154(9):4456-4465.

14. Borges L, Oliveira VK, Baik J, Bendall SC, Perlingeiro RC. Serial transplantation reveals a critical role for endoglin in hematopoietic stem cell quiescence. Blood. 2019;133(7):688-696. doi:10.1182/ blood-2018-09-874677

15. Della Porta MG, Malcovati L, Invernizzi R, et al. Flow cytometry evaluation of erythroid dysplasia in patients with myelodysplastic syndrome. Leukemia. 2006;20(4):549-555. doi:10.1038/sj. leu. 2404142

16. Cosimato V, Scalia G, Raia M, et al. Surface endoglin (CD105) expression on acute leukemia blast cells: an extensive flow cytometry study of 1002 patients. Leuk Lymphoma. 2018;59(9):2242-2245. doi:10.1080/10428194.2017.1416366

17. Poręba M, Jaźwiec B, Poręba R, Kuliczkowski K. Expression of CD105 antigen in patients with acute leukemia, malignant lymphoma, and multiple myeloma in active phase of the disease. $A d v$ Clin Exp Med. 2006;15(6):1023-1028.
18. Chakhachiro ZI, Zuo Z, Aladily TN, et al. CD105 (endoglin) is highly overexpressed in a subset of cases of acute myeloid leukemias. Am J Clin Pathol. 2013;140(3):370-378. doi:10.1309/ AJCPG8XH7ZONAKXK

19. Kauer J, Schwartz K, Tandler C, et al. CD105 (Endoglin) as negative prognostic factor in AML. Sci Rep. 2019;9(1):1. doi:10.1038/s41598019-54767-x

20. Dourado K, Baik J, Oliveira VK, et al. Endoglin: a novel target for therapeutic intervention in acute leukemias revealed in xenograft mouse models. Blood. 2017;129(18):2526-2536. doi:10.1182/blood2017-01-763581

21. Borowitz MJ, Devidas M, Hunger SP, et al.; Children's Oncology Group. Clinical significance of minimal residual disease in childhood acute lymphoblastic leukemia and its relationship to other prognostic factors: a Children's Oncology Group study. Blood. 2008;111 (12):5477-5485. PMID: 18388178; PMCID: PMC2424148. doi:10.1182/blood-2008-01-132837.

22. Pui CH, Relling MV, Sandlund JT, Downing JR, Campana D, Evans WE. Rationale and design of Total Therapy Study XV for newly diagnosed childhood acute lymphoblastic leukemia. Ann Hematol. 2004;83:S124-6. doi:10.1007/s00277-004-0850-2

23. Dawson BD, Trapp RG. Basic \& Clinical Biostatistics. 3rd ed. New York, NY: Medical Publication Division, Lange Medical Book/McGraw-Hill; 2001:161-218.

24. Xu P, Wang M, Jiang Y, Ouyang J, Chen B. The association between hypoxia inducible factor $1 \alpha$ and multidrug resistance of acute myeloid leukemia. Transl Cancer Res. 2017;6(1):198-205. doi:10.21037/ tcr.2017.01.10

25. Inaba H, Greaves M, Mullighan CG. Acute lymphoblastic leukemia. Lancet. 2013;381(9881):1943-1955. doi:10.1016/S0140-6736(12) 62187-4

26. Dias A, Kenderian SJ, Westin GF, Litzow MR. Novel therapeutic strategies in acute lymphoblastic leukemia. Curr Hematol Malig Rep. 2016;11(4):253-264. doi:10.1007/s11899-016-0326-1

27. Farhadfar N, Litzow MR. New monoclonal antibodies for the treatment of acute lymphoblastic leukemia. Leuk Res. 2016;49:13-21. doi:10.1016/j.leukres.2016.07.009
Journal of Blood Medicine

\section{Publish your work in this journal}

The Journal of Blood Medicine is an international, peer-reviewed, open access, online journal publishing laboratory, experimental and clinical aspects of all aspect pertaining to blood based medicine including but not limited to: Transfusion Medicine; Blood collection, Donor issues, Transmittable diseases, and Blood banking logistics; Immunohematology; Artificial and alternative blood based therapeutics; Hematology; Biotechnology/nanotechnology of blood related medicine; Legal aspects of blood medicine; Historical perspectives. The manuscript management system is completely online and includes a very quick and fair peer-review system. Visit http://www.dovepress.com/testimonials.php to read real quotes from published authors. 